\title{
Numerical Simulations on the Fully Developed Liquid-metal MHD Flow at High Hartmann Numbers in the Rectangular Duct
}

\author{
Xinghui Cai, Hongfu Qiang ${ }^{*}$, Sanqiang Dong, Jiangren Lu and Dianhai Wang \\ Xi'an Research Inst. Of Hi-Tech, Hongqing Town, Xi'an, 710025, P. R. China \\ ${ }^{*}$ Corresponding author
}

\begin{abstract}
Numerical simulation on the liquid metal mangetohydrodynamic (MHD) flow is a very attractive way to study the MHD flow problems. The two-level radial point interpolation method is applied to study the fully developed MHD flow in this work. With the method, numerical simulations of the MHD flows at Hartmann numbers up to $10^{4}$ in a duct with arbitrary wall conductance ratios, namely, from thin conducting to insulated wall ducts, are conducted. The non-dimensional flowrate for different electrical conductivities of the duct wall are listed and compared with the exact solutions, showing a good accuracy. These results also indicate that the higher electrical conductivity of the duct wall, the lower non-dimensional flowrate, which is consistent with the well-known MHD flow behavior. Numerical simulations for insulated walls with conducting cracks or different orientation of external applied magnetic field are also carried out with the two-level radial point interpolation method.
\end{abstract}

Keywords—MHD flow; numerical simulations

\section{INTRODUCTION}

MHD flow in the duct has been applied in many fields, such as the liquid metal flow of the cooling system of the nuclear fusion or fission reactors, MHD generators, etc. However, the MHD effect originated from the liquid metal flow in a duct under a magnetic field will cause some special effect on the flow. For the flow in the duct with ideally insulating walls, the induced currents are closed within the flow domain. However, the currents will return through the conducting walls if walls of the duct are not perfectly insulated, which will further enhance the MHD pressure drop, which is not a welcome effect in some fields. Therefore, the electrical conductivity of the wall will greatly influence the current path and the entire flow domain. The direct and effective way to reduce the MHD pressure drop is the insulation of the conducting walls. Thin layer coating has been considered as an insulating technique by some researchers [1]. However, it should be noted that the insulation properties might be reduced by corrosion, irradiation, or mechanical stress. Therefore, the influence of local damage of the coating on the flow pattern needs to be investigated.

The analytical solutions for MHD problems, except for some simple cases [2, 3], are almost impossible obtained. Therefore, numerical simulation for MHD flow problems become very important in the research. A number of numerical methods for the MHD flow in conducting or insulating ducts have been developed over the last two decades. The finiteelement method (FEM), one of the most popular numerical methods, has been employed in steady or unsteady MHD flows by B Singh et al. [4-5], M Tezer-Sezgin et al. [6] and SLL Verardi et al. [7, 8]. AI Nesiturk and M Tezer-Sezgin [9] simulated the MHD flow in a duct with the two-level FEM for the values of Hartmann number up to $10^{5}$. Stanisław K Krzemiński et al. [10] applied FEM to simulate the dynamics of magnetic field influence on a conducting liquid in a square. A Sterl [11], and N Umeda et al. [12] have carried out the MHD flow simulations under the fusion reactor blanket conditions with the finite-difference method (FDM). SK Krzeminski et al. [13] also applied FDM to obtain the numerical solutions of MHD flows inside a channel with a rapidly expanded section. A numerical code for analysis of a fully developed MHD flow in a channel of a liquid metal blanket using various insulation techniques has been developed by S Smolentsev et al. [14] using the finite-volume method.

The methods mentioned above are all based on meshes. Therefore, the quality of meshes is very important for the simulation results. Sometimes good quality meshes are very difficult to be generated. And the process of generating meshes takes much time and labor. Therefore, meshless methods are also investigated in MHD flow by many researchers.

M Tezer-Sezgin et al. applied the boundary element method to simulate the MHD flow problems in the rectangular duct [15-16] and infinite region [17]. The analytical finite element method [18] and the element-free Galerkin method (EFGM) [19] were adopted to simulate MHD flows in a duct for the Hartmann numbers less than 1000. By using the residual-free bubble functions, L Zhang et al. [20] developed a two level element-free Galerkin method and simulated the fully developed MHD flow with Hartmann numbers up to $10^{4}$.

The radial point interpolation method (RPIM), proposed in [21] and applied in [22] by JG Wang and GR Li, possess the Kronecker delta function properties, which is helpful to enforce the essential boundary conditions. However, RPIM could only simulate MHD flows [23] at lower magnetic field with Hartmann number less than 50 with out any modifications. We modified the two level element-free Galerkin method and proposed a two-level radial point interpolation method (TLRPIM)[24] to simulate MHD flow in a circular duct. In this paper, full details of TLRPIM are not repeated. The method is used to simulate MHD flow in a rectangular duct at high 
Hartmann numbers up to $10^{4}$. Numerical simulations for insulated walls with conducting cracks or different orientation of external applied magnetic field are also carried out with the two-level radial point interpolation method.

\section{The Physical PRoBlem}

For the fully developed MHD flows, all variables are independent to the axial coordinate $z$. The flow direction is a linear function of $z$. The external applied magnetic field $B_{0}$ is in the $x y$-plane and forms an angle $\alpha$ with the $y$-axis. The nondimensional equations of the fully developed MHD flow are as follows [2]:

$$
\begin{aligned}
& \nabla^{2} V+H a_{X} \frac{\partial B}{\partial X}+H a_{Y} \frac{\partial B}{\partial Y}=-1 \\
& \nabla^{2} B+H a_{X} \frac{\partial V}{\partial X}+H a_{Y} \frac{\partial V}{\partial Y}=0
\end{aligned}
$$

in the flow domain, and

$$
\frac{\partial^{2} B^{\prime}}{\partial X^{2}}+\frac{\partial^{2} B^{\prime}}{\partial Y^{2}}=0
$$

in the duct wall, where

$$
\begin{aligned}
& H a_{X}=H a \sin \alpha \\
& H a_{Y}=H a \cos \alpha \\
& H a=\sqrt{H a_{X}^{2}+H a_{Y}^{2}}
\end{aligned}
$$

The boundary conditions are

$$
V=0, B=B^{\prime} \text { and } \sigma^{\prime} \frac{\partial B}{\partial n}=\sigma \frac{\partial B^{\prime}}{\partial n}
$$

on the boundary interface between the fluid and the wall; and the induced magnetic filed

$$
B_{2}=0
$$

on the outside boundary of the duct. $\sigma$ and $\sigma^{\prime}$ are the electric conductivity of the fluid and the wall, respectively. $B$ and $B^{\prime}$ are the non-dimensional induced magnetic field of the fluid and the duct walls, respectively. $V$ is the nondimensional velocity of the fluid. $n$ is the unit normal vector to the boundary of the flow section. Ha is the Hartmann number. The non-dimensional parameters in the above equations are

$$
\begin{gathered}
X=\frac{x}{a}, Y=\frac{y}{a}, B=-\frac{B_{z}}{\left(a^{2} / \eta\right)(\partial p / \partial z) \mu_{0} \sqrt{\eta \sigma}}, \\
V=-\frac{v_{z}}{\left(a^{2} / \eta\right) \partial p / \partial z}, H a=B_{0} a \sqrt{\frac{\sigma}{\eta}}
\end{gathered}
$$

where $\eta$ is the fluid viscosity coefficient. $v_{z}(x, y)$, $B_{z}(x, y)$ and $p$ are the axial velocity, the induced magnetic field and the pressure, respectively. $\mu_{0}$ is a constant, which has the value $4 \pi \times 10^{-7}$ in the MKS system. $a$ is the characteristic length of the flow section.

Suppose that the thickness $h$ of the duct wall be much smaller than the characteristic dimension $a$, which is a limiting case analyzed by Shercliff [3], then the full problem reduces to solve (1) and (2) in the flow domain, with the boundary conditions:

$$
V=0
$$

$$
\frac{\partial B}{\partial n}+\theta B=0
$$

where $\theta=\frac{\sigma^{\prime} h}{\sigma a}$.

\section{NUMERICAL SIMULATIONS}

\section{A. Rectangular Duct}

As many numerical methods could simulate MHD flow at low or moderate Hartmann numbers, the results for Hartmann numbers less than 500 are not presented in this paper again.

Shercliff analyzed the MHD flow with non-conducting walls and gave the analytical results [3]. The velocity field of the numerical simulation for $\alpha=\pi / 2, H a=10^{4}$ is presented in Figure 1 (a). The comparison between numerical and Shercliff's analytical results at $X=0$ is shown in Figure 1 (b).
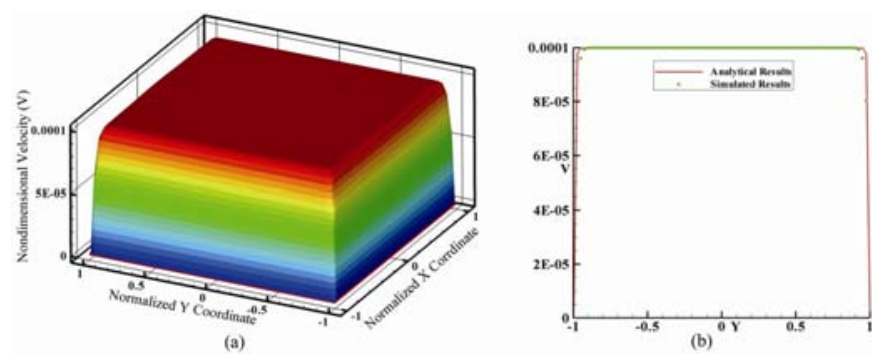

FIGURE I. VELOCITY PROFILE (a) AND COMPARISON BETWEEN NUMERICAL AND SHERCLIFF'S ANALYTICAL RESULTS AT $X=0$ (B) FOR $\alpha=\pi / 2, H a=10000$

Analytical solutions for the MHD flows in a rectangular duct with conducting walls were obtained by JCR Hunt [2]. The velocity profiles and the comparisons between numerical 
and Hunt's analytical solutions for $\theta=10, \alpha=\pi / 2, H a=10^{3}$ are shown in Figure 2 (a) and (b), respectively.

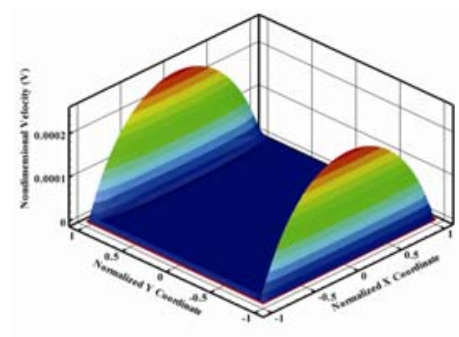

(a)

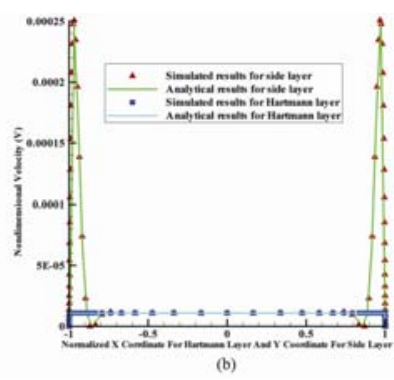

FIGURE II. VELOCITY PROFILE (A) AND COMPARISON BETWEEN NUMERICAL AND HUNT'S ANALYTICAL RESULTS (B) AT HARTMANN LAYER AND SIDE LAYER FOR $\theta=10, \alpha=\pi / 2, H a=1000$

The non-dimensional flowrate can be achieved with the formula $Q=\iint V(X, Y) d X d Y$. The solutions of nondimensional flowrate for different value of $\theta$ from TLRPIM and analytical method are both presented in Table 1.

TABLE I. COMPARISON BETWEEN TLRPIM'S AND HUNT'S FLOW RATE OF DIFFERENT $\Theta$ FOR $H A=1000$

\begin{tabular}{|c|r|r|r|r|r|c|}
\hline $\boldsymbol{\Theta}$ & $\mathbf{0}$ & $\mathbf{0 . 0 0 1}$ & $\mathbf{0 . 0 0 5}$ & $\mathbf{0 . 0 1}$ & $\mathbf{0 . 0 5}$ & $\mathbf{0 . 5}$ \\
\hline TLRPIM & $\begin{array}{c}3.90 \times 10^{-} \\
3\end{array}$ & $\begin{array}{r}2.20 \times 10^{-} \\
3\end{array}$ & $\begin{array}{r}7.73 \times 10^{-} \\
4\end{array}$ & $\begin{array}{r}4.45 \times 10^{-} \\
4\end{array}$ & $\begin{array}{r}1.35 \times 10^{-} \\
4\end{array}$ & $\begin{array}{c}5.82 \times 10^{-} \\
5\end{array}$ \\
\hline Hunt & $\begin{array}{r}4.10 \times 10^{-} \\
3\end{array}$ & $\begin{array}{r}2.19 \times 10^{-} \\
3\end{array}$ & $\begin{array}{r}7.65 \times 10^{-} \\
4\end{array}$ & $\begin{array}{r}4.40 \times 10^{-} \\
4\end{array}$ & $\begin{array}{r}1.33 \times 10^{-} \\
4\end{array}$ & $\begin{array}{c}5.69 \times 10^{-} \\
5\end{array}$ \\
\hline
\end{tabular}

From the above figures and Table 1, it can be seen that the numerical results of velocity and non-dimensional flowrates with TLRPIM match the analytical results pretty well.

\section{B. Coated Duct with Cracks}

As mentioned above, small cracks in the insulating coating will influence the flow pattern in the duct. Researchers have done some investigations on this problem [25], [26]. The method may also simulate this problem through the change of the magnetic field boundary conditions. Several cases of the cracks in the coating of rectangular duct are presented in Figure 3.
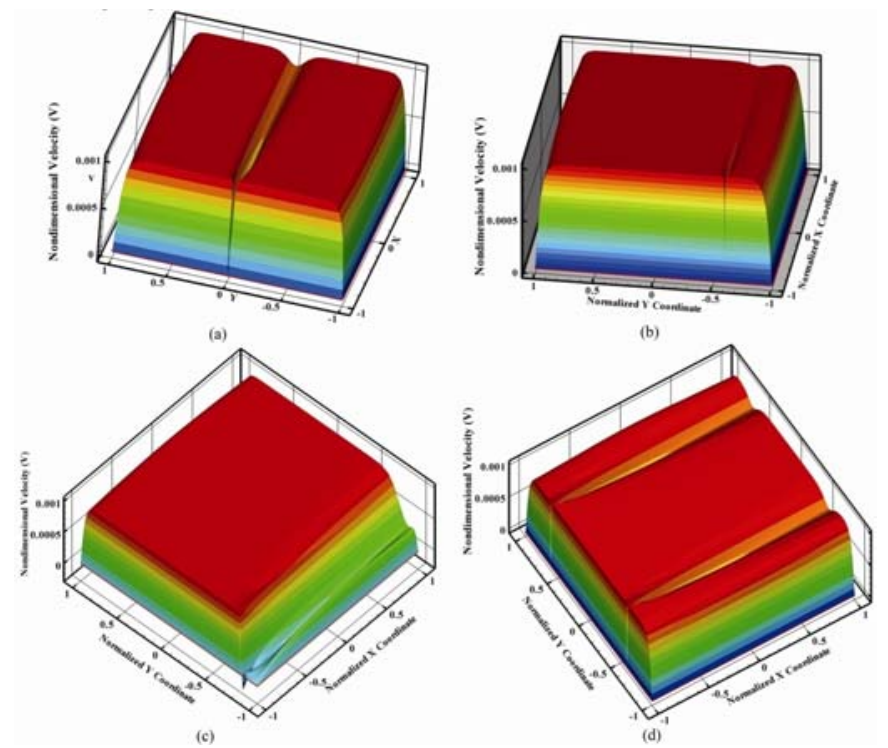

FIGURE III. VELOCITY FIELD PROFILES FOR $H a=1000, \Theta=10, \alpha=\pi / 2$, LOCATION OF THE CRACK: $\mathrm{X}=-1$, (A) $-0.01 \leq \mathrm{Y} \leq 0.01$ (B) $-0604 \leq \mathrm{Y} \leq-0.599$ (C) $1 \leq \mathrm{Y} \leq-0.8$ (D) $-0.61 \leq \mathrm{Y} \leq-0.59,0.59 \leq \mathrm{Y} \leq 0.61$

TABLE II. THE INFLUENCE OF THE LOCATION AND SIZE OF CRACKS ON THE FLOWRATE

\begin{tabular}{|c|c|c|c|}
\hline Location and range & Flowrate & Location and range & Flowrate \\
\hline With out crack & $3.901 \times 10^{-3}$ & $-1 \leq \mathrm{y} \leq-0.98, \mathrm{x}=-1$ & $3.88 \times 10^{-3}$ \\
\hline $\mathbf{- 0 . 0 1} \leq \mathbf{y} \leq \mathbf{0 . 0 1}, \mathbf{x}=-\mathbf{1}$ & $3.824 \times 10^{-3}$ & $-0604 \leq \mathrm{y} \leq-0.599, \mathrm{x}=-1$ & $3.864 \times 10^{-3}$ \\
\hline $\mathbf{- 0 . 6 1} \leq \mathbf{y} \leq \mathbf{- 0 . 5 9}, \mathbf{x}=-\mathbf{1}$ & $3.817 \times 10^{-3}$ & $-1 \leq \mathrm{y} \leq-0.8, \mathrm{x}=-1$ & $3.597 \times 10^{-3}$ \\
\hline $\mathbf{- 0 . 7 1} \leq \mathbf{y} \leq \mathbf{- 0 . 6 9 ,}, \mathbf{x}=-\mathbf{1}$ & $3.834 \times 10^{-3}$ & $\begin{array}{c}-0.61 \leq \mathrm{y} \leq-0.59, \\
0.59 \leq \mathrm{y} \leq 0.61, \mathrm{x}=-1\end{array}$ & $3.759 \times 10^{-3}$ \\
\hline $\mathbf{- 0 . 8 1} \leq \mathbf{y} \leq \mathbf{- 0 . 7 9 ,}, \mathbf{x}=-\mathbf{1}$ & $3.836 \times 10^{-3}$ & $-0604 \leq \mathrm{x} \leq-0.599, \mathrm{y}=-1$ & $3.90 \times 10^{-3}$ \\
\hline
\end{tabular}

Table 2 presents the numerical results of non-dimensional flowrate for the insulated walls of a duct with some local cracks. As seen in table 2, the number, size, and location of the cracks will influence the flowrate. Less number or smaller size of the cracks will cause less reduction of the flowrate. For the cracks of same size, if the distance of the crack location is further to the center of the Hartmann layer, the effect to reduce the flowrate is smaller. However, the center of the Hartmann layer is not the location where the smallest flowrate appears. If the crack is located on the side layer, it does not affect the flowrate at all.

\section{Influence of the Angle of External Applied Magnetic Field}

If the external applied magnetic field is not perpendicular or parallel to the walls of the duct but forms an oblique angle with them, the velocity and magnetic filed will change greatly. The results of velocity profiles for $75^{\circ}, 60^{\circ}$, and $45^{\circ}$, insulated walls and conducting walls of $\theta=10, H a=1000$ are presented in Figures 4 and 5, respectively. 


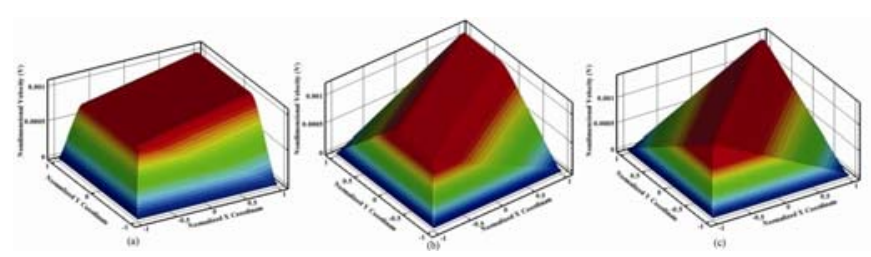

FIGURE IV. VELOCITY PROFILES FOR $H a=1000$, (a) $\alpha=75^{\circ}$, (b) $\alpha=60^{\circ}$, AND (c) $\alpha=45^{\circ}$, INSULATED WALLS

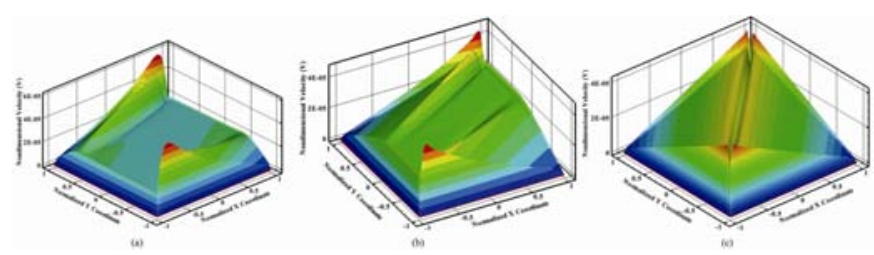

FIGURE V. VELOCITY FIELD PROFILES FOR $H a=1000$, (a) $\alpha=75^{\circ}$, (b) $\alpha=60^{\circ}$, AND (c) $\alpha=45^{\circ}, \theta=10$

\section{CONCLUSIONS}

A two-level radial point interpolation method is used to simulate the fully developed MHD flow in several cases. With TLRPIM, the method may be applied to the MHD flow in a duct with conducting or insulating walls. The numerical results for Shercliff's and Hunt's cases from the TLRPIM were compared with the analytical solutions. The results have shown a good accuracy for both conducting and non-conducting walls at high Hartmann numbers. Numerical simulations for MHD flows in the duct with the conducting wall cracks and in the cases that the external applied magnetic field forming an oblique angle with the walls of the rectangle duct were also performed with good stability.

\section{REFERENCES}

[1] D. L. Smith, J. H. Park, I. Lyublinski, V. Evtikhin, A. Perujo, H. Glassbrenner, T. Terai, and S. Zinkle, "Progress in coating development for fusion systems," Fusion Eng. Des. vol. 61, pp. 629-641, 2002.

[2] J. C. R. HUNT, "Magnetohydrodynamic flow in rectangular ducts," J. Fluid. Mech. vol. 21, pp. 577-590, 1965

[3] J. A. Shercliff, "Steady motion of conducting fluids in pipes under transverse magnetic fields," Math. Proc. Cambridge. vol. 49, pp.136-144, 1953.

[4] B.Singh and J. Lal, "Finite element method in magnetohydrodynamic channel flow problems" Int. J. Numer. Meth. Eng. vol. 18, pp. 1104$1111,1982$.

[5] B. Singh and J. Lal, "Finite element method for unsteady MHD flow through pipes with arbitrary wall conductivity," Int. J. Numer.l Meth. vol. 4, pp291-302, 1984.

[6] M. Tezer-Sezgin and S. Köksal, "Finite element method for solving MHD flow in a rectangular duct", Int. J. Numer. Meth. Eng. vol. 28, pp445-459, 1989.

[7] S. L. L. Verardi, J. R. Cardoso and C. C. Motta, "A solution of twodimensional magnetohydrodynamic flow using the finite element method", IEEE T. Magn. vol. 34, pp. 3134-3137, 1998.

[8] S. L. L. Verardi, J. R. Cardoso and M. C. Costa, "Three-dimensional finite element analysis of MHD duct flow by the penalty function formulation”, IEEE T. Magn. vol. 37, pp. 3384-3387, 2001.

[9] A. I. Nesiturk and M. Tezer-Sezgin, "The finite element method for MHD flow at high Hartmann numbers", Comput. Method. Appl. Mech. Eng. vol. 194, pp. 1201-1224, 2005.

[10] K. Stanisław Krzemiński Michał Śmiałek and Maciej Włodarczyk, "Finite Element Approximation of Biharmonic Mathematical Model for
MHD Flow Using $\psi$-an approach”, IEEE T. Magn. vol. 36, pp. 1313$1318,2000$.

[11] A. Sterl, "Numerical simulation of liquid-metal MHD flows in rectangular ducts", J. Fluid. Mech. vol. 216, pp161-191,1990.

[12] N. Umeda and M. Takahashi, "Numerical analysis for heat transfer enhancement of a lithium flow under a transverse magnetic field", Fusion Eng. Des., vol. 51/52, pp. 899-907, 2000.

[13] S. K. Krzeminski, A. Cala and M. Smialek, "Numerical simulation of 2D MHD flows $\psi-\xi-$ A method", IEEE Trans. Magn. vol. 32, pp. 990993,1996.

[14] S. Smolentsev, N. B. Morley and M. Abdou, "Code development for analysis of MHD pressure drop reduction in a liquid metal blanket using insulation technique based on a fully developed flow model", Fusion Eng. Des. vol. 73, pp. 83-93, 2005.

[15] C. Bozkaya and M. Tezer-Sezgin, "Boundary element solution of unsteady magnetohydrodynamic duct flow with differential quadrature time integration scheme", Int. J. Numer. Meth. Fl. vol. 51, pp. 567-584, 2006.

[16] M. Tezer-Sezgin and C. Bozkaya, "Boundary element method solution of magnetohydrodynamic flow in a rectangular duct with conducting walls parallel to applied magnetic field", Comput. Mech. vol. 41, pp. 769-775.

[17] M. Tezer-Sezgin and C. Bozkaya, "The boundary element solution of the magnetohydrodynamic flow in an infinite region", J. Comput. Appl. Math. vol.225, pp. 510-521, 2009.

[18] Z. Demendy and T. Nagy, "A new algorithm for solution of equations of MHD channel flows at moderate Hartmann numbers" Acta Mech. vol. 123, pp.135-149, 1997.

[19] S. L. L. Verardi, J.M. Machado and J. R. Cardoso, "The element-free Galerkin method applied to the study of fully developed magnetohydrodynamic duct flows", IEEE Trans. Magn. vol. 38, pp. 941-944, 2002.

[20] L. Zhang, Jie O.Y. and X. H. Zhang, "The two-level element free Galerkin method for MHD flow at high Hartmann numbers", Phys. Lett. A, vol. 372, pp. 5625-5638, 2008.

[21] J. G. Wang and G. R. Liu, "A point interpolation meshless method based on radial basis functions", Int. J. Numer. Meth. Eng. vol. 54, pp. 1623$1648,2002$.

[22] J. G. Wang and G. R. Liu, "On the optimal shape parameters of radial basis functions used for 2-D meshless methods", Comput. Method. Appl. Mech. Eng. vol. 191, pp. 2611-2630, 2002.

[23] X. H. Cai, G.H. Su and S. Z. Qiu, "Local radial point interpolation method for the fully developed magnetohydrodynamic flow", Appl. Math. Comput. vol. 217, pp. 4529-4539,2011.

[24] E. J. Kansa, "Multiquadrics-A scattered data approximation scheme with application to computational fluid dynamics", Comput. Math. Appl. vol. 19, pp.147-161,1990.

[25] A. Ying and A. Gaizer, "The effects of imperfect insulating coatings on MHD and heat transfer in rectangular ducts", Fusion Eng. Des. vol. 27 pp. $634-41,1995$.

[26] L. Buhler, "The influence of small cracks in insulating coatings on the flow structure and pressure drop in MHD channel flows", Fusion Eng . Des. vol. 27, pp650-658,1995. 